к. э. н., доцент, доиент кафедры «Экономическая теория и эконометрика», Институт управления, экономики и финансов, Казанский федеральный университет, e-mail: kad-ekaterina@yandex.ru

БАКИРОВА АЙСЫЛУ НАРИМОВНА

Высшая школа информационных технологий и интеллектуальных систем,

Казанский федеральный университет, e-mail: $a b @ i t . k f u . r u$

\author{
АБДУЛАЕВ ХАВАЖ-БАУДИ САЛАУДЫЕВИЧ \\ Институт управления, экономики и финансов, факультет экономики, \\ Казанский федеральный университет, \\ e-mail: Islamabdulaev1998@mail.ru \\ ДРОЗДОВА ДАРЬЯ АЛЕКСЕЕВНА \\ Институт управления, экономики и финансов, факультет экономики, \\ Казанский федеральный университет, \\ e-mail: dashunkadrozdova@icloud.com
}

\title{
ЭКОНОМИЧЕСКИЙ РОСТ В РЕГИОНАХ: ФАКТОРЫ И ТЕНАЕНЦИИ
}

Аннотауия. Џель работы. Оиенка влияния объёма инвестииий в основной капитал и затрат на технологические инновации на валовой региональный продукт, измеряющий экономический оост. Метод ияи методология проведения работы. На основе обзора теорий экономического роста акиентировано внимание на источниках регионального экономического роста. Применение линейной модели множественной регрессии позволило оценить методом наименьших квадратов регрессанты регионального экономического роста, выполнить сравнение их доверительных интервалов, рассчитать частные коэффициенты эластичности. Результаты. Моделирование с 2010 по 2016 годы в отдельности позволило проследить динамику происходящих изменений в регрессанте под влиянием регрессоров. С помощью частных коэффиииентов эластичности сделан вывод о степени влияния факторов на результативный показатель. Вывод о статистически значимом различии во влиянии объемов инвестииий в основной капитал и затрат на технологические инновации на валовой региональный продукт формулируется на основе сравнения модульных значений границ доверительных интервалов: если интервалы пересекаются, то статистически нет различий между коэффициентами. Область применения результатов. Представляется возможным рекомендовать применить полученные результаты региональным органам власти при разработке региональной экономической политики в области привеления инвестииий и внедрения инноващий. Выводы. Эмпирически подтверждено предположение о том, что рост объема инвестииий в основной капитал и рост затрат на технологические инновации увеличивают произведенный валовой региональный продукт. Инвестииии в основной капитал оказывают более значительное воздействие на валовой региональный продукт, чем затраты на технологические инновации. В 2015, 2016 годы по сравнению с 2010, 2011, 2012, 2013 годами статистически различно влияние инвестиций в основной капитал в сторону увеличения, относительно влияния затрат на технологические инноващии. В дальнейших исследованиях для устранения смещения в оценках коэффициентов регрессии иелесообразно расширить круг регрессоров валового регионального продукта, применять эконометрические модели анализа панельных данHolx.

Ключевые слова: валовой внутренний продукт, инвестищии в основной капитал, регрессионный анализ, линейная модель множественной регрессии. 
KADOCHNIKOVA EKATERINA IVANOVNA

$\rho_{h D}$ Econ., associate professor, associate professor "Economic theory and econometrics", Institute of management, economy and finance, Kazan Federal University, e-mail:kad-ekaterina@yandex.ru

BAKIROVA AYSYLU NARIMOVNA

The higher school of information technologies and intellectual systems, Kazan Federal University, e-mail: ab@it.kfu.ru

ABDULLAEV HAVAZH-BAUDI SALAUDYEVICH

Institute of management, economy and finance, faculty of economy, Kazan Federal University, e-mail: Islamabdulaev1998@mail.ru

DROZDOVA DARYA ALEKSEEVNA

Institute of management, economy and finance, faculty of economy, Kazan Federal University, e-mail: dashunkadrozdova@icloud.com

\title{
ECONOMIC GROWTH IN REGIONS: FACTORS AND TRENDS
}

\begin{abstract}
Work purpose. Assessment of the impact of the volume of investment into fixed capital and costs for technology innovations for the gross regional product measuring economic growth. Method or methodology of carrying out work. On the basis of the overview of theories of economic growth the attention is focused on sources of regional economic growth. Application of linear model of multiple regression allowed to evaluate the least-squares method regressant of regional economic growth, to execute comparison of their confidential intervals, to calculate private coefficients of elasticity. Results. Modeling from 2010 to 2016 separately allowed to track dynamics of the happening changes in a regressant under the influence of regressors. By means of private coefficients of elasticity the conclusion is drawn on extent of influence of factors on a productive indicator. A conclusion about statistically significant difference in influence of the volumes of investment into fixed capital and costs for technology innovations for a gross regional product is formulated on the basis of comparison of modular values of borders of confidential intervals: if intervals are crossed, then statistically there are no differences between coefficients. Scope of results. It is obviously possible to recommend to apply the received results to regional authorities when developing regional economic policy in the field of a priveleniye of investments and introduction of innovations. Conclusions. The assumption that growth of the volume of investment into fixed capital and growth of costs for technology innovations increases the made gross regional product is empirically confirmed. Investments into fixed capital make more considerable impact on a gross regional product, than costs for technology innovations. In 2015, 2016 in comparison with 2010, 2011, 2012, 2013 statistically variously influence of investments into fixed capital towards increase, concerning influence of costs for technology innovations. In further researches for elimination of shift in estimates of regression coefficients it is expedient to expand a circle of regressors of a gross regional product, to apply econometric models of the analysis of panel data.
\end{abstract}

Keywords: gross internal product, investments into fixed capital, regression analysis, linear model of multiple regression.

Введение. Изучение регионального экономического роста включает как минимум три аспекта: рост производства, рост потребления и координация обоих. Вопрос о возможности достижения экономического роста путем инвестиций до сих пор остается дискуссионным в экономической науке. В свое время А. Смит указал такие ключевые источники роста как разделение труда, накопление капитала и технический прогресс. В современной цифровой экономике этим источникам роста большое внимание уделяют в развитии современных 
теорий, исследуя процесс преобразования экономических факторов роста, таких как технический прогресс и человеческий капитал, в эндогенные факторы. В модели ХарродаДомара $[13,14]$ использована производственная функция, в которой количество капитала и труда, потребляемых при производстве одной единицы продукта являются фиксированными и экзогенными. В неоклассической модели экономического роста Солоу-Свена $[19,20]$ факторы экономического роста взаимозаменяемы, с переменным технологическим коэффициентом капитал и труд автоматически приспосабливаются к сбалансированному росту. Хотя модель Солоу-Свена рассматривает технический прогресс как основной, фактически единственный экзогенный фактор для роста экономики в долгосрочной перспективе. Согласно Ф. Найт, Ф. Рамсей, Р. Солоу без технологических изменений рост инвестиций не может привести к долгосрочному экономическому росту. Новые теории роста предложили модели с эндогенным фактором технического прогресса. Кеннет Дж. Эрроу [12] предположил, что технический прогресс или рост производительности является побочным продуктом накопления капитала, то есть эффектом, вызванным инвестициями и накоплением человеческого капитала за счет обучения. В результате Эрроу показал технический прогресс как эндогенную переменную, определяемую экономической системой. В 1980-х годах П. Ромер [16], Ф. Агион и П. Ховитт [11] представили эндогенную теорию экономического роста, основанную на распространении знаний среди производителей в результате преднамеренных исследований и разработок. Поэтому технологический прогресс является одновременно выражением накопления человеческого капитала и улучшения качества инвестированного капитала. Внутреннее качество инвестированного капитала повышается с ростом уровня технологий, созданных посредством использования человеческого капитала. Помимо накопления человеческого капитала, углубление специализации капитала также может способствовать углублению разделения труда и формированию монопольного конкурентного преимущества, что делает экономический рост более стабильным и устойчивым и приводит к увеличению предельной доходности инвестированного капитала. Тем самым в настоящее время теории экономического роста развиваются вокруг трех аспектов [15]: технологические инновации, продвижение которых способствует углублению разделения труда; накопление человеческого капитала, которое прямо выражается через технический прогресс; материализация технологий в инновации, которые проявляются в накоплении капитала.

Опираясь на вышеизложенный обзор теорий роста, в качестве основных источников регионального экономического роста можно назвать технический прогресс, инвестиции, накопление человеческого капитала посредством технического прогресса, накопление качества капитала посредством технологий. В свою очередь технологии можно разделить на четыре категории: повышение качества и технологии новых продуктов, технологии снижения затрат, технологии управления и общие операционные технологии. Повышение качества капитала приведет к общему технологическому прогрессу в сфере производства и в сфере потребления. Обзор теорий экономического роста позволили нам сформулировать цель работы - на примере экономики России проверить предположение о том, что рост объёма инвестиций в основной капитал и затрат на технологические инновации увеличивают произведённый валовой региональный продукт, измеряющий экономический рост.

Методы исследования. В исследовании по данным сборника по данным сборника «Регионы России. Социально-экономические показатели. 2017.» оценена линейная модель множественной регрессии, которая обеспечивает четкую экономическую интерпретацию рассматриваемых параметров и позволяет по заданным значениям регрессоров находить теоретические значения регрессанта $[2,3,6]$ :

$$
Y_{i}=\beta_{0}+\beta_{1} X_{i 1}+\beta_{2} X_{i 2}+\varepsilon_{i}
$$

где, $\mathrm{Y}_{\mathrm{i}}$ - валовой региональный продукт, тыс. руб., $\mathrm{X}_{\mathrm{i} 1}$ - объем инвестиций в основной капитал, тыс. руб., $X_{\text {i2 }}$ - затраты на технологические инновации, тыс. руб., $\beta_{0}, \beta_{1}, \beta_{2}$, - параметры регрессии, оцененные обычным методом наименьших квадратов, $\varepsilon$ - случайное отклонение (погрешность). Для сопоставимости показателей расчеты производились на душу населения, согласно численности населения в регионах. 
Основная цель множественной регрессии - построить модель с несколькими регрессантами, определив при этом влияние каждого из них в отдельности, а также совокупное воздействие их на моделируемый показатель. Классический подход к оцениваю параметров линейной регрессии основан на методе наименьших квадратов. Метод наименьших квадратов позволяет получить такие оценки параметров регрессии $\beta_{\mathrm{j}}$, при которых сумма квадратов отклонений фактических значений регрессанта $y$ от расчетных (теоретических) $y_{x}$ минимальна, при этом параметры регрессии $\beta_{\mathrm{j}}$ являются частными производными регрессанта $y$ по соответствующим факторам $x_{j}$.

Результаты. Целесообразность включения регрессоров $\mathrm{X}_{\mathrm{i} 1}, \mathrm{X}_{\mathrm{i} 2}$ во множественную регрессию подтверждается проведенным с помощью инструмента «Корреляция» в Microsoft Excel корреляционным анализом (табл.1)

Таблииа 1

Результаты корреляционного анализа регрессоров ВРП в 2016 году

\begin{tabular}{|c|c|c|c|}
\hline & $Y$ & $X 1$ & $X 2$ \\
\hline $\mathrm{Y}$ & 1 & & \\
\hline $\mathrm{X} 1$ & 0,85 & 1 & 1 \\
\hline $\mathrm{X} 2$ & 0,48 & 0,12 & \\
\hline
\end{tabular}

Согласно шкале Чеддока - Снедекора наблюдается тесная прямая статистическая взаимосвязь между произведенным ВРП и объемом инвестиций в основной капитал $\left(\mathrm{R}_{\mathrm{yx}}=\right.$ 0,85), а также умеренная прямая статистическая взаимосвязь между произведенным ВРП и затратами на технологические инновации $\left(\mathrm{R}_{\mathrm{yx} 2}=0,48\right)$. При этом статистическая взаимосвязь между факторами $\left(\mathrm{R}_{\mathrm{x} 1 \times 2}=0,12\right)$ практически отсутствует, что свидетельствует о соблюдении одного из требований к построению множественной регрессии: в уравнение включаются регрессоры, которые между собой не взаимосвязаны.

Полученные с помощью инструмента «Регрессия» в Microsoft Excel результаты регрессионного анализа (табл. 2) отражают высокое качество линейной модели множественной регрессии. Коэффициент детерминации показывает, что построенная регрессия объясняет $86,93 \%$ вариации произведенного в регионе ВРП. P-value для статистики Фишера в нашем случае составило $1,81 \mathrm{E}-25$, что меньше, чем $\alpha=0,01$, это означает, что с вероятностью $99 \%$ отвергается нулевая гипотеза о неадекватности уравнения регрессии. Согласно тесту Фишера регрессия адекватна, выбранный набор регрессоров оказывает линейное влияние на регрессант Ү - ВРП.

Для проверки гипотезы о значимости каждого из коэффициентов множественной регрессии воспользуемся $\mathrm{t}$ - статистикой Стьюдента. В нашем случае $p$-value статистики Стьюдента для коэффициента регрессии при $\mathrm{X}_{1}$ составило $5 \mathrm{E}-25$, что меньше, чем $\alpha=0,01$. Это означает, что коэффициент регрессии $\beta_{1}-$ значим с вероятностью $99 \%$, между переменными $\mathrm{X}_{1}$ (объем инвестиций в основной капитал) и $\mathrm{Y}$ (ВРП) существует значимая линейная связь. Также $p$-value статистики Стьюдента для коэффициента регрессии при $\mathrm{X}_{2}$ составило 0,002 , что меньше, чем $\alpha=0,01$. Это означает, что коэффициент регрессии $\beta_{2}-$ значим с вероятностью $99 \%$, между переменными $\mathrm{X}_{2}$ (затраты на технологические инновации) и $\mathrm{Y}$ (ВРП) существует значимая линейная связь. Свободный коэффициент $\alpha$ является значимым с вероятностью 99\% (P-value статистики Стьюдента составило 3Е-07, что меньше, чем $\alpha=0,01)$. Границы доверительных интервалов значение «ноль» не включают, подтверждая значимость коэффициентов уравнения регрессии.

Но для того, чтобы проследить динамику происходящих изменений необходима более расширенная выборка рассматриваемых параметров, поэтому нами была использована выборка с официального сайта Федеральной службы государственной статистики из 83 регионов в период с 2010 по 2016 годы (рассматриваемые показатели остались теми же). Полученные результаты представлены в таблице 3 . 
РЕГИОНАЛЬНЫЕ ПРОБЛЕМЫ ПРЕОБРАЗОВАНИЯ ЭКОНОМИКИ, №12, 2018

Результаты регрессионного анализа влияния объема инвестиций

в основной капитал и затрат на технологические инновации на произведенный ВРП по субъектам России в 2016 году

\begin{tabular}{|c|c|c|c|c|c|c|c|c|}
\hline \multicolumn{9}{|c|}{ Регрессионная статистика } \\
\hline \multicolumn{4}{|c|}{ Множественный R } & \multicolumn{5}{|c|}{0,8714} \\
\hline \multicolumn{4}{|l|}{ R-квадрат } & \multicolumn{5}{|c|}{0,8693} \\
\hline \multicolumn{4}{|c|}{ Нормированный R-квадрат } & \multicolumn{5}{|c|}{0,7533} \\
\hline \multicolumn{4}{|c|}{ Стандартная ошибка } & \multicolumn{5}{|c|}{374} \\
\hline \multicolumn{4}{|l|}{ Наблюдения } & \multicolumn{5}{|c|}{83} \\
\hline \multicolumn{9}{|c|}{ Дисперсионный анализ } \\
\hline & $d f$ & $S S$ & $M S$ & $F$ & \multicolumn{4}{|c|}{ Значимость $F$} \\
\hline Регрессия & 2 & $3,5 \mathrm{E}+07$ & $2 \mathrm{E}+07$ & 126,2 & \multicolumn{4}{|c|}{$1,81 \mathrm{E}-25$} \\
\hline Остаток & 80 & $1,1 \mathrm{E}+07$ & 140067 & & & & & \\
\hline \multirow[t]{2}{*}{ Итого } & 82 & $4,7 \mathrm{E}+07$ & & & & & & \\
\hline & $\begin{array}{c}\text { Коэффииии- } \\
\text { ентьь }\end{array}$ & $\begin{array}{l}\text { Стандарт- } \\
\text { ная ошибка }\end{array}$ & $\begin{array}{c}t- \\
\text { статистика }\end{array}$ & Р-Значение & $\begin{array}{c}\text { Нижние } \\
95 \%\end{array}$ & $\begin{array}{c}\text { Верхние } \\
95 \%\end{array}$ & $\begin{array}{c}\text { Нижние } \\
95,0 \%\end{array}$ & $\begin{array}{c}\text { Верхние } \\
95,0 \%\end{array}$ \\
\hline Y-пересечение & 291 & 51,6047 & 5,6332 & $3 \mathrm{E}-07$ & 188,00 & 393,4 & 188 & 393 \\
\hline $\mathrm{X} 1$ & 0,46 & 0,03031 & 15,063 & $5 \mathrm{E}-25$ & 0,3962 & 0,517 & 0,396 & 0,52 \\
\hline $\mathrm{X} 2$ & 15,4 & 4,76643 & 3,2354 & 0,002 & 5,9357 & 24,91 & 5,935 & 24,9 \\
\hline
\end{tabular}

Таблица 3

Значение коэффициентов эластичности и доверительных интервалов

\begin{tabular}{|c|c|c|c|}
\hline Год & Уравнение множественной регрессии & $\begin{array}{l}\text { Коэффициенты } \\
\text { эластичности }\end{array}$ & $\begin{array}{c}\text { Доверительные интервалы для } \\
\text { коэффициентов регрессии }\end{array}$ \\
\hline 2010 & $Y_{x}=142,97+0,266 X_{1}+21,52 X_{2}$ & $\begin{array}{l}Э_{1}=0,26 \\
Э_{2}=0,01\end{array}$ & $\begin{array}{l}98,92 * * *<\alpha<187,02 * * * \\
0,24 * * *<\beta_{1}<0,29 * * * \\
13,37 * * *<\beta_{2}<29,68 * * *\end{array}$ \\
\hline 2011 & $\mathrm{Y}_{\mathrm{x}}=174,22+0,304 \mathrm{X}_{1}+17,31 \mathrm{X}_{2}$ & $\begin{array}{l}Э_{1}=0,24 \\
Э_{2}=0,23\end{array}$ & $\begin{array}{l}114,55^{* * *}<\alpha<233,89^{* * *} \\
0,27^{* * *}<\beta_{1}<0,34^{* * *} \\
9,25^{* * *}<\beta_{2}<25,37^{* * *}\end{array}$ \\
\hline 2012 & $Y_{x}=186,20+0,23 X_{1}+21,12 X_{2}$ & $\begin{array}{l}Э_{1}=0,18 \\
Э_{2}=0,31\end{array}$ & $\begin{array}{l}114,46^{* * *}<\alpha<257,94 * * * \\
0,19 * * *<\beta_{1}<0,28 * * * \\
11,24 * * *<\beta_{2}<31,01 * * *\end{array}$ \\
\hline 2013 & $Y_{x}=227,09+0,31 X_{1}+12,62 X_{2}$ & $\begin{array}{l}Э_{1}=0,22 \\
Э_{2}=0,20\end{array}$ & $\begin{array}{l}151,24 * * *<\alpha<302,95 * * * \\
0,28^{* * *}<\beta_{1}<0,36^{* * *} \\
5,54 * * *<\beta_{2}<19,72^{* * *}\end{array}$ \\
\hline 2014 & $Y_{x}=248,98+0,35 X_{1}+13,59 X_{2}$ & $\begin{array}{l}Э_{1}=0,22 \\
Э_{2}=0,21\end{array}$ & $\begin{array}{l}162,51^{* * *}<\alpha<335,45^{* * *} \\
0,29^{* * *}<\beta_{1}<0,41^{* * *} \\
5,92^{* * *}<\beta_{2}<21,27 * * *\end{array}$ \\
\hline 2015 & $Y_{x}=279,55+0,41 X_{1}+13,64 X_{2}$ & $\begin{array}{l}Э_{1}=0,24 \\
Э_{2}=0,18\end{array}$ & $\begin{array}{l}184,01^{* * *}<\alpha<375,12 * * * \\
0,36^{* * *}<\beta_{1}<0,47 * * * \\
5,3 * * *<\beta_{2}<21,98^{* * *}\end{array}$ \\
\hline 2016 & $\mathrm{Y}_{\mathrm{x}}=290,102+0,46 \mathrm{X}_{1}+15,42 \mathrm{X}_{2}$ & $\begin{array}{l}Э_{1}=0,24 \\
Э_{2}=0,20\end{array}$ & $\begin{array}{l}188,01 * * *<\alpha<393,4 * * * \\
0,39 * * *<\beta_{1}<0,52 * * * \\
5,94 * * *<\beta_{2}<24,91 * * *\end{array}$ \\
\hline
\end{tabular}

Примечание: ***, **, *-значимость на уровне 1\%, 5\%, и 10\% соответственно. 
Рассчитанные показатели, представленные в таблице 3, позволяют сделать вывод не только о наличии линейной зависимости между исследуемыми факторами, но также объясняют их влияние и динамику. Так, например, по рассчитанным коэффициентам регрессии за 2016 год можем утверждать, что при увеличении объема инвестиций в основной капитал на 1 ден. ед., объем ВРП увеличивается на 0,46 ден. ед., а если затраты на технологические инновации увеличить на 1 ден. ед., то произведенный ВРП увеличится на 15,42 ден. ед.

С помощью полученных частных коэффициентов эластичности можно сделать вывод о степени влияния факторов на результативный показатель. Так, в 2016 году увеличение объемов инвестиций в основной капитал на $1 \%$ привело к увеличению ВРП на $0,24 \%$, а увеличение затрат на технологические инновации на $1 \%$ привело к увеличению ВРП на $0,20 \%$. Динамика частных коэффициентов эластичности за 2010 - 2016 год показывает, что инвестиции в основной капитал оказывают более значительное воздействие на ВРП, чем затраты на технологические инновации (исключение 2012 год, где Э $\succ_{1} \ni_{2}$ ).

Построив уравнение множественной регрессии для расчета зависимого параметра Y произведенный ВРП, мы можем прогнозировать лишь его приблизительное значение, поскольку существует случайная ошибка. Поэтому, чтобы оценить фактические значения рассматриваемых параметров, необходимо рассчитать доверительные интервалы коэффициентов регрессии (табл. 3). По данным 2016 года доверительные интервалы для коэффициентов регрессии составляют: $188,01<\alpha<393,4 ; 0,39<\beta 1<0,52 ; 5,94<\beta 2<24,91$. Заметим, что диапазон границ доверительного интервала для коэффициента регрессии желательно не должен превышать 3 . В нашем случае, для коэффициентов $\alpha$ и $\beta_{1}$ это условие выполняется (правая граница больше левой в 2,09 и 1,33 раза соответственно), а для коэффициента $\beta_{2}$ это значение превышает установленный максимум и составляет 4,19 раза. Такая тенденция для доверительных интервалов коэффициентов регрессии прослеживается во всех рассматриваемых временных промежутках. Это говорит о том, что фактическое влияние коэффициента $\beta_{2}$ при переменной $X_{2}$ (затраты на технологические инновации) варьируется в больших промежутках, нежели фактические значения для $\alpha$ и $\beta$. Поэтому качество подгонки рекомендуется улучшить.

Вывод о статистически значимом различии во влиянии объемов инвестиций в основной капитал и затрат на технологические инновации на валовой региональный продукт предлагается формулировать на основе сравнения модульных значений границ доверительных интервалов: если интервалы пересекаются, то статистически нет различий между коэффициентами. Так, в 2015,2016 годы по сравнению с 2010, 2011, 2012, 2013 годами статистически различно влияние инвестиций в основной капитал в сторону увеличения относительно влияния затрат на технологические инновации. Статистически различное влияние затрат на технологические инновации на валовой региональный продукт не выявлено, модульные значения границ доверительных интервалов пересекаются во всех попарных сравнениях. Полученные в работе результаты согласуются с выводами, сформулированными в работах $[1,4,5,7,9,10]$.

Выводы. Таким образом, согласно проведенному анализу, предположение о том, что рост объема инвестиций в основной капитал и рост затрат на технологические инновации увеличивают произведенный ВРП получило эмпирическое подтверждение. Рассчитанные коэффициенты регрессии и их доверительные интервалы, а также частные коэффициенты эластичности характеризуют степень влияния факторов на результативный показатель и определяют границы её вариации. Проведенный регрессионный анализ углубляет представления о характере исследуемой взаимосвязи и более точно доказывает статистическую значимость выбранных показателей.

Поскольку инвестиции в основной капитал и затраты на технологические инновации являются не единственными факторами, влияющими на ВРП, а следовательно, и экономический рост регионов, в дальнейших исследованиях для устранения смещения в оценке коэффициентов регрессии при переменных в модель целесообразно включить дополнительные регрессоры валового регионального продукта. Для оценки влияния факторов на экономический рост в долгосрочной перспективе рекомендуется применять эконометрические модели анализа панельных данных. 
Лumepamypa

1. Бувальцева В.И., Федулова Е.А., Альбина Т.А. Оиенка реализации инвестиционной стратегии территории как целевого ориентира устойчивого экономического роста на основе корреляционнорегрессионного анализа // Экономика и сочиальная политика. №6, С. 9-15. 2014.

2. Демидова, О. А. Эконометрика: учебник и практикум для прикладного бакалавриата / О. А. Демидова, Д. И. Малахов. - М.: Издательство Юрайт, 2018. - 334 с. - Серия: Бакалавр. Прикладной курс.

3. Елисеева И.И., Курышева С.В., Костеева Т.В. и др. Эконометрика: Учебник / Под ред. И.И. Елисеевой. - 2-е изд., перераб. и доп. - М.: Финансы и статистика, 2005. - 576 с.

4. Кольцов Р.Ю., Кольчова О.В. Корреляиионно-регрессионный анализ и прогноз основньх показателей развития рынка региона (на примере Тамбовской области) // Вестник Тамбовского университета. Серия «Естественные и технические науки». № 2. С. 669-672. 2016.

5. Кондратьев Н.В. Экономический анализ взаимосвязи инвестиций и экономического роста // Финансы и бизнес. № 4. С. 21-34. 2010.

6. Новиков А.И. Эконометрика: Учебное пособие, 3-е изд.// - М.: НИЦ «ИНФРА-М». 2011. 272 c.

7. Пидямев О.П. Оиенка инвестиций в основной капитал в современных условиях // Econотісs: Yesterday, Today and Tomorrow. №7. C. 170-179. 2016.

8. Сборник «Регионы России. Соииально-экономические показатели. 2017.». - М. Федеральная служба государственной статистики, 2017.

9. Ущеев Ф.А., Чиркова С.С. Инвестициии, экономический рост и конвергенциия в России и в мире: эконометрический подход // Финансы и бизнес. № 1. С. 41-51. 2008.

10. Чалик А.В., Мудревский А.Ю. Корреляциионно-регрессионный анализ валового регионального продукта // Международный научно - исследовательский журнал. № 6. C.52-56. 2017.

11. Aghion P., Howitt P. A model of growth through creative destruction. Econometrica., V.60(2), P.323351. 1992

12. Arrow K. J. The economic implications of learning-by-doing. Review Economic Study. V.9, P.155-173. 1962.

13. Domar E. D. Capital expansion, rate of growth, and employment. Econometrica. V.14, P.137-147. 1946.

14. Harrod R. An essay in dynamic theory. Economic Journal. V. 49, P.14-33. 1939.

15. M. Liang, The Microeconomic Growth, Springer-Verlag, Berlin, Heidelberg. 2014.

16. Romer P.M. Endogenous technological change. Journal Polit Economic. V. 98(5) Part 2: P.71-102. 1990.

17. Romer P.M. Increasing returns and long-run growth. Journal Polit Economic. V.94(5), P. 1002-1037. 1986.

18. Romer P.M. The Origins of Endogenous Growth. The Journal of Economic Perspectives. Vol.8, Iss. 1. P. 3-22. 1994.

19. Solow R. M. A contribution to the theory of economic growth. Quart Journal Economic V.70(1). P.8694. 1956.

20. Swan T. W. Economic growth and capital accumulation. Economic Rec. V.32, P. 334-361. 1956.

\section{References:}

1. Aghion P., Howitt P. A model of growth through creative destruction. Econometrica., V.60(2), P.323351. 1992

2. Anthology "Regions Of Russia. Socio-economic indicators. 2017.." - M. Federal state statistics service, 2017.

3. Arrow K. J. The economic implications of learning-by-doing. Review Economic Study. V.9, P.155-173. 1962.

4. Buvaltseva V. I., Fedulova E. A., Albina T. A. Evaluation of the investment strategy of the territory as a target of sustainable economic growth on the basis of correlation analysis. No. 6, pp. 9-15. 2014.

5. Chalik A.V., Mudrewski A.Yu. Correlation and regression analysis gross regional product // International research journal. No. 6. pp. 52-56. 2017.

6. Demidova, O. A. Econometrics: textbook and practical course for applied baccalaureate / O. A. Demidova, D. I. Malakhov. - Moscow: Yurayt Publishing House, 2018. - 334 p. - Series: Bachelor. Application course.

7. Domar E. D. Capital expansion, rate of growth, and employment. Econometrica. V.14, P.137-147. 1946.

8. Eliseeva I. I., Kurysheva S.V., Kasteeva T. V. etc. Econometrics: Textbook/ Under the editorship of I. I. Eliseeva. - 2nd ed., edited by - M.: Finance and statistics, 2005. - 576 p.

9. Evaluation of investments in fixed assets in modern conditions // Economics: Yesterday, Today and Tomorrow.No. 7. pp. 170-179. 2016.

10. Harrod R. An essay in dynamic theory. Economic Journal. V. 49, P.14-33. 1939.

11. Koltsov, O. V. Koltsova O.V. Correlation and regression analysis and forecast of the main indicators of the regional market development (on the example of the Tambov region). Vestnik of the Tambov University. Series Natural and technical Sciences."No. 2. pp. 669-672. 2016.

12. Kondratyev N. V. Economic analysis of the relationship of investment and economic growth// Finance and business. No. 4. pp. 21-34. 2010.

13. M. Liang, The Microeconomic Growth, Springer-Verlag, Berlin, Heidelberg. 2014.

14. Novikov A.I. Econometrics: textbook, 3rd ed./ Moscow: National Research Center I'NFRA-M." 2011.272

$p$. 
КАДОЧНИКОВА Е.И., БАКИРОВА А.Н., АБДУЛАЕВ Х-Б.С., ДРОЗДОВА Д.А. ЭКОНОМИЧЕСКИЙ РОСТ В РЕГИОНАХ: ФАКТОРЫ И ТЕНДЕНЦИИ

15. Romer P.M. Endogenous technological change. Journal Polit Economic. V. 98(5) Part 2: P.71-102. 1990.

16. Romer P.M. Increasing returns and long-run growth. Journal Polit Economic. V.94(5), P. 1002-1037. 1986.

17. Romer P.M. The Origins of Endogenous Growth. The Journal of Economic Perspectives. Vol.8, Iss. 1. P. 3-22. 1994.

18. Solow R. M. A contribution to the theory of economic growth. Quart Journal Economic V.70(1). P.8694. 1956

19. Swan T. W. Economic growth and capital accumulation. Economic Rec. V.32, P. 334-361. 1956.

20. Uschev F.A. Chirkova S.S. Investment, economic growth and convergence in Russia and in the world: an econometric approach / Finance and business. No. 1. pp $41-51$. 2008. 